\title{
Ferromagnetism of Nanosized Bilayer on Square Lattice Within Ising Model: Static and Dynamic Aspects with Size Effects Using Pair Approximation
}

\author{
Ö. GÖRGÜLÜER ${ }^{a}$, O. YALÇIN ${ }^{a, *}$ AND R. ERDEM ${ }^{b}$ \\ ${ }^{a}$ Department of Physics, Niğde Ömer Halisdemir University, 51240 Niğde, Turkey \\ ${ }^{b}$ Department of Physics, Akdeniz University, 07058 Antalya, Turkey
}

Received: 01.07.2020 \& Accepted: 22.12.2020

Doi: 10.12693/APhysPolA.139.109 *e-mail: o.yalcin@ohu.edu.tr

\begin{abstract}
In this study, the pair approximation in the Kikuchi version is applied to study ferromagnetism of a nanosized bilayer system on a square lattice using the Ising model. Each layer is divided into the core, core-surface and surface parts as introduced for the monolayers by Özüm et al. (2015). Temperature, magnetic field and interlayer exchange energy variations of the long- and short-range order parameters are investigated. Some second-order phase transitions between ferromagnetic and paramagnetic phases with size effects are observed. By using the path probability method, the flow of the system towards some stable equilibrium states in the ferromagnetic phase is obtained via relaxation curves and flow diagrams. The results of the numerical calculations are in a fairly good agreement with the available theoretical works of other authors.
\end{abstract}

topics: nanosized bilayer system, pair approximation, interlayer exchange energy, static and dynamic properties

\section{Introduction}

The magnetic multilayer systems have potential applications in information storage, retrieval magnets, spintronics, optoelectronic devices, stored energy and giant magnetoresistance devices [1-6]. Many studies, such as those of spin-valve read heads, magnetic random access memories and magnetic switch devices, have focused on bilayer ferromagnets. They are the first stage between a two-dimensional magnetic plane and a threedimensional bulk magnet. In general, the number of magnetic atoms in each layer of bilayers and their orientation under the external magnetic field $h$ are related to the technological significance of these systems. The Ising model and its variants are very useful tools in the study of the magnetic properties of such bilayer systems [7-42]. The authors of this paper construct some different types of lattice structures, such as a triangular lattice [25, 28], square lattice [8], honeycomb lattice [36-39], a Bethe lattice $[16,26,27]$, as well as graphene-like structures $[29,30,42]$. Interestingly, second-order phase transitions were presented in some bilayer studies $[16,23,26]$ as well. The static and dynamic properties of magnetization for square lattice bilayer systems have been investigated so far using a variety of techniques such as the pair approximation [19], self-consistent field theory [20], effective field theory [21, 24], Monte-Carlo simulation [31], dynamic mean-field theory [33] and Glauber-type stochastic dynamics [34, 35]. However, a parallel study of both the static and dynamic properties of short-range order parameter $Q$ with magnetization $M$ for square lattice bilayer systems has not been performed in detail for such systems, e.g., for those with different layer sizes and interlayer interactions.

In the scope of this work, we have investigated the layer size effects, second-order phase transition, interlayer interaction effects and long- and short-range orders that are controlled by the interlayer interaction in the bilayer system via static and dynamics properties with different sizes. We study the static properties under the pair approximation (PA) [43] while for its dynamics we use the path probability method (PPM) with pair distribution [44]. The PPM is also known as the natural extension into the time domain of the PA. This particular method has been proved to have the potential to provide information on both the equilibrium and the non-equilibrium properties of the spin systems [45-48]. In the current study, we examined a spin system at an equilibrium state. Moreover, for the same system at nonequilibrium, $M$ vs. time $t$ and $Q$ vs. $t$ curves (or relaxation curves), as well as the flow diagrams in the phase space of $M$ vs. $Q$ are discussed in the context of some geometrical factors of each layer in the system. 


\section{Ö. Görgülüer et al.}

The paper is organized as follows: Sect. 2 is devoted to the presentation of the spin system and a short description of the used methods, the PA and the PPM, in Sect. 3, we present and discuss the calculated results and Sect. 4 offers concluding remarks on salient features of the current investigation.

\section{Theoretical model}

A schematic representation of a nanosized bilayer system on the square lattice $(a b)$ is shown in Fig. 1. Each layer in 2D exhibits three shells of spins with $L_{a}=L_{b}=3$. Hence, the size of each layer increases as either $L_{a}$ or $L_{b}$ increases. The filled-circles in red and blue colors correspond to the core $C$ and surface $S$ spins in the layers, respectively. The filledcircles contain a core spin number $\left(N_{C}^{a}, N_{C}^{b}\right)$, a coresurface $(C S)$ spin number $\left(N_{C S}^{a}, N_{C S}^{b}\right)$ and a surface spin number $\left(N_{S}^{a}, N_{S}^{b}\right)$. The total number of spins $\left(N^{a}, N^{b}\right)$ in a bilayer system covers the spins of layers $a$ and $b$ with $N=N^{a}+N^{b}, N^{a}=N_{C}^{a}+N_{S}^{a}$, $N^{b}=N_{C}^{b}+N_{S}^{b}$, and $N^{a b}=\left(N^{a}+N^{b}\right) / 2$. Further, each layer is described by core spins $\left(S_{a}^{C}\right.$ and $\left.S_{b}^{C}\right)$, core-surface spins $\left(S_{a}^{C S}\right.$ and $\left.S_{b}^{C S}\right)$ and surface spins $\left(S_{a}^{S}\right.$ and $\left.S_{b}^{S}\right)$

In the presence of external magnetic field $h$, Hamiltonian for the above spin system can be expressed as

$$
\begin{array}{r}
H=-J^{a} \sum_{\langle i j\rangle} S_{i} S_{j}-J^{b} \sum_{\langle i j\rangle} \sigma_{i} \sigma_{j} \\
-J^{a b} \sum_{\langle i j\rangle} S_{i} \sigma_{j}-h \sum_{\langle i j\rangle}\left(S_{i}+\sigma_{j}\right),
\end{array}
$$

where $J^{a}$ and $J^{b}$ are the intralayer bilinear exchange coupling interactions between nearest-neighbor atoms (denoted by $\langle i j\rangle$ ) with spins $S_{i}= \pm 1$ in layer $a$ and $\sigma_{i}= \pm 1$ in layer $b$, respectively, while $J^{a b}$ denotes the interlayer bilinear exchange coupling. All the exchange coupling parameters appearing in (1) are given in $k_{\mathrm{B}} T$ units where $k_{\mathrm{B}}$ is the Boltzmann constant and $T$ is the temperature. The cases of $J^{a}>0, J^{b}>0, J^{a b}>0$ and $J^{a}<0$, $J^{b}<0, J^{a b}<0$ correspond to ferromagnetic (FM) and antiferromagnetic (AFM) interactions, respectively. A paramagnetic (PM) phase exists in the system when $J^{a}=J^{b}=J^{a b}=0$ for all temperatures. The fractions of the spin states $( \pm 1)$ are called the point (or state) variables $X_{i}$. In the PA method developed by Kikuchi [43], other variables, known as the bond (or pair) variables $Y_{i j}$, are introduced. If the total number of spin pairs in the system is $N_{p}$, the number of $(+1,+1)$ bonds is $Y_{11} N_{p}$, the number of $(+1,-1)$ bonds is $Y_{12} N_{p}$ $\left(Y_{12}=Y_{21}\right)$ whereas the number of $(-1,-1)$ bonds is $Y_{22} N_{p}$. The relations between point variables and bond variables is given by $X_{i}=\sum_{j=1}^{2} Y_{i j}(i=1,2)$.

We now separate (1) into three terms $\left(H_{C}, H_{C S}, H_{S}\right)$, including all interactions between nearest neighboring spins in core regions $\left(J_{C}^{a}, J_{C}^{b}\right)$, interfaces $\left(J_{C S}^{a}, J_{C S}^{b}\right)$ and surface parts
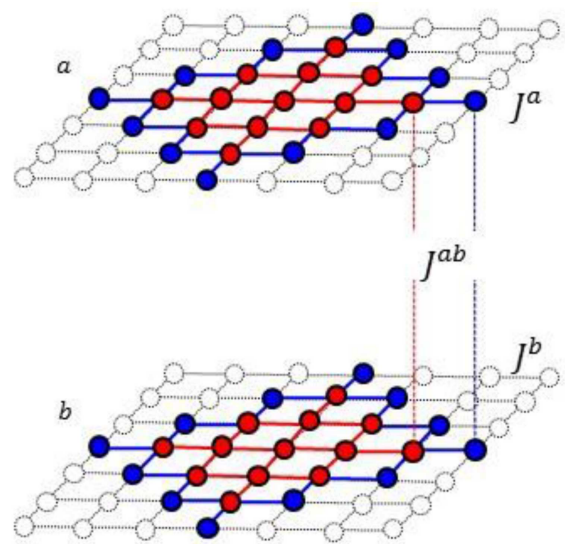

Fig. 1. A schematic view of a square lattice bilayer system with three shells $\left(L_{a}=L_{b}=3\right)$. The upper and lower layers containing the spins labeled as $S_{a}$ and $\sigma_{b}$ are marked as $a$ and $b$. The intralayer bilinear exchange coupling interactions between nearest-neighbor atoms and the interlayer bilinear exchange coupling, respectively, are denoted as $J^{a}, J^{b}$ and $J^{a b}$.

$\left(J_{S}^{a}, J_{S}^{b}\right)$ of the layers. The explicit details of the above formulation are presented for a nanoscale monolayer in [49-53]. By making use of the definitions from [45], a long-range order parameter $M$, i.e., average magnetization and a short-range order parameter $Q$ are calculated, respectively

$$
\begin{aligned}
& M=X_{1}-X_{2}=Y_{11}-Y_{22}, \\
& Q=Y_{11}-2 Y_{12}+Y_{22} .
\end{aligned}
$$

Here, the bond variables are found from the numerical solutions of the set of equations:

$$
Y_{i j}=\frac{1}{Z}\left(X_{i} X_{j}\right)^{(\gamma-1) / \gamma} \mathrm{e}^{-\beta \varepsilon_{i j}} \equiv Z^{-1} e_{i j},
$$

where $i, j=1,2, \beta=\left(k_{\mathrm{B}} T\right)^{-1}$ and $\gamma$ is the coordination number of a given lattice site inside the bilayer system and $Z$ is the partition function defined by the relation $Z=\sum_{i, j=1}^{2} e_{i j}$. For the energy parameters $\varepsilon_{i j}$ in (4), one introduces the following definition:

$$
\varepsilon_{i j}=\varepsilon_{i j}^{a}+\varepsilon_{i j}^{a b}+\varepsilon_{i j}^{b}, \quad i, j=1,2,
$$

where

$$
\begin{aligned}
& \varepsilon_{i j}^{a}=N_{p, C}^{a} \varepsilon_{i j, C}^{a}+N_{p, C S}^{a} \varepsilon_{i j, C S}^{a}+N_{p, S}^{a} \varepsilon_{i j, S}^{a}, \\
& \varepsilon_{i j}^{a b}=N^{a b} \gamma^{a b} \varepsilon^{a b}, \\
& \varepsilon_{i j}^{b}=N_{p, C}^{b} \varepsilon_{i j, C}^{b}+N_{p, C S}^{b} \varepsilon_{i j, C S}^{b}+N_{p, S}^{b} \varepsilon_{i j, S}^{b},
\end{aligned}
$$

Here, one reads

$$
\begin{aligned}
N_{p, C}^{a} & =\frac{1}{2} N_{C}^{a} \gamma_{C}^{a}-N_{C S}^{a}, \quad N_{p, C S}^{a}=N_{C S}^{a} \gamma_{C S}^{a}, \\
N_{p, S}^{a} & =\frac{1}{2} N_{S}^{a} \gamma_{S}^{a}
\end{aligned}
$$

and

$$
\begin{aligned}
N_{p, C}^{b} & =\frac{1}{2} N_{C}^{b} \gamma_{C}^{b}-N_{C S}^{b}, \quad N_{p, C S}^{b}=N_{C S}^{b} \gamma_{C S}^{b}, \\
N_{p, S}^{b} & =\frac{1}{2} N_{S}^{b} \gamma_{S}^{b}
\end{aligned}
$$


TABLE I

Numbers of the spins and spin pairs within the $C, C S$ and $S$ regions.

\begin{tabular}{l|c|c|c|c|c|c}
\hline \hline \multicolumn{1}{c|}{$L_{a}=L_{b}$} & 2 & 3 & 4 & 5 & 6 & 7 \\
\hline$N_{C}^{a}=N_{C}^{b}$ & 5 & 13 & 25 & 41 & 61 & 85 \\
$N_{S}^{a}=N_{S}^{b}$ & 8 & 12 & 16 & 20 & 24 & 28 \\
$N_{C S}^{a}=N_{C S}^{b}$ & 6 & 10 & 14 & 18 & 22 & 26 \\
$N_{p, C}^{a}=N_{p, C}^{b}$ & 4 & 16 & 36 & 64 & 100 & 144 \\
$N_{p, C S}^{a}=N_{p, C S}^{b}$ & 12 & 20 & 28 & 36 & 44 & 52
\end{tabular}

TABLE II

Bond energies of spin pairs $i, j$ for $C, C S$ and $S$ ions of $a$ and $b$ layers.

\begin{tabular}{c|c|c|c|c}
\hline \hline Layers & Pair & Core & $\begin{array}{c}\text { Core- } \\
\text { surface }\end{array}$ & Surface \\
\hline \multirow{2}{*}{$a$} & $\varepsilon_{11}^{a}$ & $-J_{C}^{a}-2 h$ & $-J_{C S}^{a}$ & $-J_{S}^{a}-2 h$ \\
& $\varepsilon_{12}^{a}=\varepsilon_{21}^{a}$ & $J_{C}^{a}$ & $J_{C S}^{a}$ & $J_{S}^{a}$ \\
$\varepsilon_{22}^{a}$ & $-J_{C}^{a}+2 h$ & $-J_{C S}^{a}$ & $-J_{S}^{a}+2 h$ \\
\hline \multirow{2}{*}{$b$} & $\varepsilon_{11}^{b}$ & $-J_{C}^{b}-2 h$ & $-J_{C S}^{b}$ & $-J_{S}^{b}-2 h$ \\
& $\varepsilon_{12}^{b}=\varepsilon_{21}^{b}$ & $J_{C}^{b}$ & $J_{C S}^{b}$ & $J_{S}^{b}$ \\
& $\varepsilon_{22}^{b}$ & $-J_{C}^{b}+2 h$ & $-J_{C S}^{b}$ & $-J_{S}^{b}+2 h$ \\
\hline Interlayer & $\varepsilon^{a b}$ & \multicolumn{3}{|c}{$-J^{a b}$} \\
\end{tabular}

as the numbers of spin pairs in each layer. These variables are listed in Table I. The coordination numbers are assumed to be $\gamma_{C}^{a}=\gamma_{C}^{b}=4, \gamma_{C S}^{a}=$ $\gamma_{C S}^{b}=2, \gamma_{S}^{a}=\gamma_{S}^{b}=0, \gamma^{a b}=2$, while $\varepsilon_{i j, C}^{a}, \varepsilon_{i j, C S}^{a}$, $\varepsilon_{i j, S}^{a}, \varepsilon_{i j, C}^{b}, \varepsilon_{i j, C S}^{b}, \varepsilon_{i j, S}^{b}$ are called the bond energies of spin pairs $i j$ for $C, C S, S$ ions of layers $a$ and $b$, respectively [49-53], and $\varepsilon^{a b}$ is the interlayer bond energy for the neighboring spins. Their numerical values are computed by using (1), as shown in Table II. The three nonlinear algebraic equations, namely (4), are solved by using the NewtonRaphson or iteration method for a fixed layer size $L_{a}=L_{b}$ and a given value of $T, h$, and $J^{a b}$. After establishing the $Y_{i j}$ values, the $M$ and $Q$ values can be obtained easily by using (2) and (3), respectively. In the next section, we shall examine the thermal, magnetic field and interlayer exchange variations of the system at equilibrium for different layer sizes.

The time dependence of $M$ and $Q$, dynamic (or rate) equations for the bilayer system, has been obtained by using the PPM [44]. The rate of change of the bond variables is given by [45-48]:

$$
\begin{gathered}
\frac{\mathrm{d} Y_{i j}}{\mathrm{~d} t}=-k_{1} \sum_{k l=\text { trans }}\left(P_{i j, k l}-P_{k l, i j}+P_{i j, l k}-P_{l k, i j}\right) \\
-k_{2} \sum_{k l=\text { rot }}\left(P_{i j, k l}-P_{k l, i j}+P_{i j, l k}-P_{l k, i j}\right),
\end{gathered}
$$

where $P_{i j, k l}$ is the path probability rate for the system to go from state $i j$ to $k l$ and $k_{1}$ and $k_{2}$ are the rate constants. The detailed balance of the system requires that

$$
P_{i j, k l}=P_{k l, i j}
$$

for all $i j, k l$. Using the Recipe II equation of Kikuchi [44], the path probability rate can be written as

$$
P_{i j, k l}=Z^{-1} e_{k l} Y_{i j},
$$

where $e_{k l}$ is found by using (4). Now, inserting (11) to $(9)$, the rate equations for the bond variables are obtained

$$
\begin{aligned}
& \frac{\mathrm{d} Y_{11}}{\mathrm{~d} t}=k_{1} Z^{-1}\left(e_{11} Y_{12}-e_{12} Y_{11}\right) \\
& \frac{\mathrm{d} Y_{12}}{\mathrm{~d} t}=\frac{1}{2} Z^{-1} e_{12}\left(k_{1} Y_{11}+k_{2} Y_{22}\right) \\
& -\frac{1}{2} Z^{-1}\left(k_{1} e_{11}+k_{2} e_{22}\right) Y_{12} \\
& \frac{\mathrm{d} Y_{22}}{\mathrm{~d} t}=k_{1} Z^{-1}\left(e_{22} Y_{12}-e_{12} Y_{22}\right)
\end{aligned}
$$

Using (2), (3) and (12), the dynamic equations for the order parameters can be expressed in the form [45]:

$$
\begin{aligned}
& \frac{\mathrm{d} M}{\mathrm{~d} t}=\frac{\mathrm{d} Y_{11}}{\mathrm{~d} t}-\frac{\mathrm{d} Y_{22}}{\mathrm{~d} t}, \\
& \frac{\mathrm{d} Q}{\mathrm{~d} t}=\frac{\mathrm{d} Y_{11}}{\mathrm{~d} t}-2 \frac{\mathrm{d} Y_{12}}{\mathrm{~d} t}+\frac{\mathrm{d} Y_{22}}{\mathrm{~d} t} .
\end{aligned}
$$

The above nonlinear equations have been solved numerically by using the Runge-Kutta method and the solutions have been plotted as the relaxation curves of $M$ and $Q$ and the flow diagrams in the two-dimensional $M-Q$ phase space for fixed values of layer size, $T, h, J^{a b}, k_{1}$ and $k_{2}$.

\section{Results and discussion}

In this section, we investigate the static and dynamic aspects of long- and short-range order parameters $(M, Q)$ for the square lattice bilayer system within the Ising model depicted in Fig. 1. The schematic illustrations of thermal, interlayer exchange coupling (with $J^{a b}>0$ and $J^{a b}<0$ ) and magnetic field behaviors for $M$ and $Q$, as well as their time evolutions are presented in Figs. 2-11. Especially, we have examined the flow diagrams in a phase space of $M$ vs. $Q$ and relaxation curves for the time-dependence properties. For simplicity, we have chosen the FM interaction energies among the spins in the core region and across the $C S$ interface inside the layers with $J_{C}^{a}=J_{C}^{b}=1$ and $J_{C S}^{a}=J_{C S}^{b}=1$. We then present the AFM case $\left(J_{C S}^{a}=J_{C S}^{b}=-1\right.$, as dashed lines) in one figure (see Fig. 2) for a comparison with the FM case (solid lines). In the above calculations, there is no contribution to the energy parameters in (5) and (6) coming from the exchange interactions among the spins in the surface part since the lattice coordination number of the surface part is zero, i.e., $\gamma_{S}^{a}=\gamma_{S}^{b}=0$. Hence, we have considered the cases with $J_{S}^{a}=J_{S}^{b}=0$.

For the static properties, we first draw a graph of long- and short-range order parameters vs. temperature $T$ in the absence of external magnetic field. 

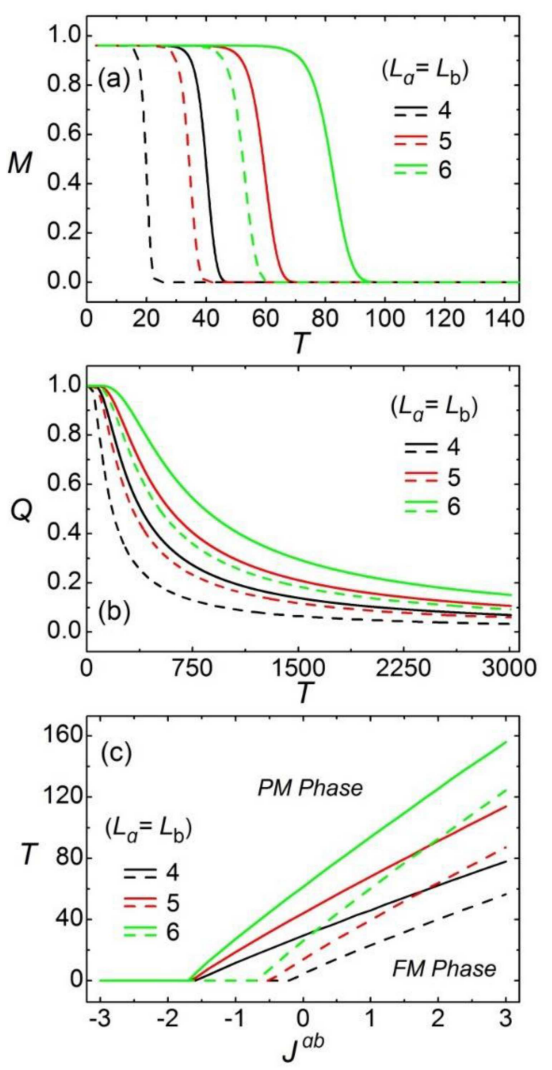

Fig. 2. (a) Temperature $T$ dependence of $M$ at various layer sizes for $h=0$ and $J^{a b}=1$ with their second-order phase transition, (b) the same as (a) but for $Q$, (c) corresponding phase diagram in the $T-J^{a b}$ plane. The solid lines and dashed lines indicate FM and AFM interactions, respectively.

The results using three different values of layer sizes with $L_{a}=L_{b}=4,5,6$ shells are given in Fig. 2 for the chosen $J^{a b}=1.0$. Figure 2 a can be used to determine the points where the second-order phase transition occurs. We observe that $M$ and $Q$ become saturated as $M \rightarrow 0.9616$ and $Q \rightarrow 1.0$ when $T$ approaches zero while they decrease continuously with increasing temperature and one of them, $M$, converges to zero, as seen in Fig. 2a and b. This behavior evidently refers to a continuous (or second-order) phase transition from the ferromagnetic phase to the paramagnetic phase at zero magnetic field, as seen in Fig. 2a. As stated in our previous study [50], the first-order phase transitions are observed in the presence of biquadratic interaction $K$ and single ion anisotropy $D$ in the nanosized structures. The transition temperatures are strongly dependent on the layer size and proportional to $L_{a}\left(=L_{b}\right)$. We also see in Fig. $2 \mathrm{c}$ that critical temperature values $T_{C}$ decrease for decreasing values of $J^{a b}$ in the $J^{a b}>0$ regime. With a further decrease in $J^{a b}$ below the $J^{a b}=0$ value, $T_{C}$ continues to decrease and eventually becomes zero. On the other hand, to determine the effects of $J_{C S}^{a}\left(=J_{C S}^{b}\right)$ on the critical temperatures, we refer to the predictions of (2) and (3) for the dependence

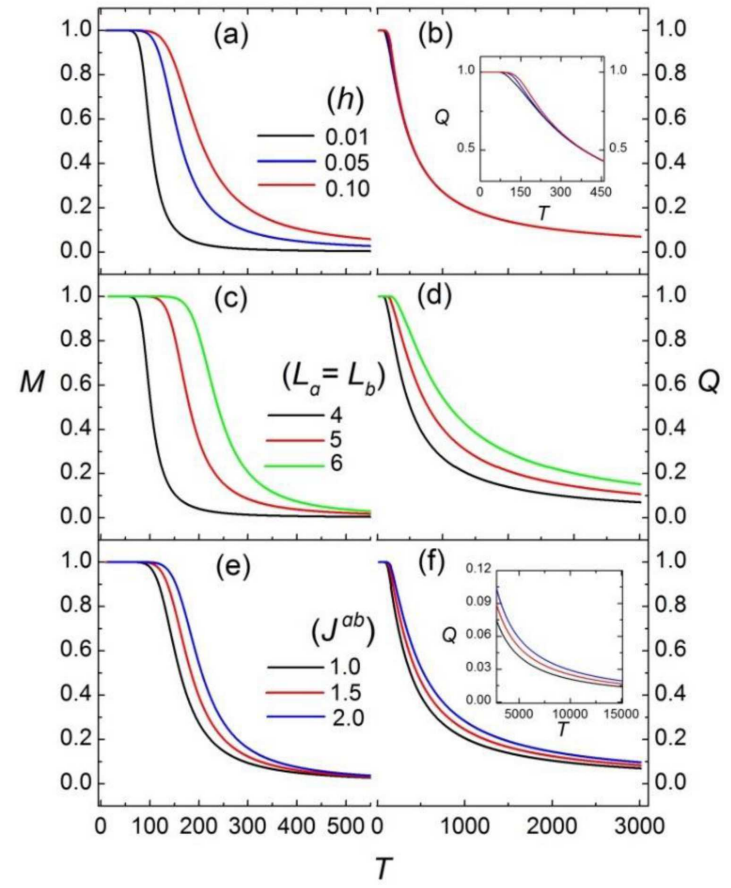

Fig. 3. (a) Temperature $T$ dependence of $M$ at various field values for $L_{a}=L_{b}=4$ and $J^{a b}=1$, (b) the same as (a) but for $Q$, (c) $M$ vs. $T$ at various layer sizes for $h=0.01$ and $J^{a b}=1$, (d) the same as (c) but for $Q$, (e) $M$ vs. $T$ at various interlayer interactions for $L_{a}=L_{b}=4$ and $h=0.05$, (f) the same as (e) but for $Q$. Note that $J^{a b}>0$.

of $M$ and $Q$ on temperature using $J_{C S}^{a}=J_{C S}^{b}=-1$. Our calculations predict that the positions of the curves are different when the interaction energy between spins in the $C$ and $S$ parts is changed, e.g., when $C$ and $S$ spins exhibit the AFM interaction $M$ vs. $T$ and $Q$ vs. $T$ curves shifted to lower temperatures (see the dashed curves in Fig. 2a and b). Thus, the AFM exchange coupling causes a decrease in the critical temperatures (dashed curves in Fig. 2c).

In Figs. 3 and $4, M$ vs. $T$ and $Q$ vs. $T$ are plotted for nonzero field values $(h \neq 0)$. We obtained the curves for three different values of the magnetic field, $L_{a}\left(=L_{b}\right)$ and $J^{a b}$. According to Fig. 3a and b, $M$ and $Q$ decrease continuously from one towards zero as the temperature increases and hence the phase transition has been removed in the presence of external magnetic field. Magnetization decreases to zero, however, at a lower temperature than a short-range order parameter. If the bilayer size is increased, both $M$ and $Q$ also grow as shown in Fig. 3c and d, respectively. Similarly, for any temperature, increasing the FM interlayer interaction energy with $J^{a b}>0$ causes an increase in the order parameters (Fig. 3e and f). In order to examine the temperature dependence of both order parameters for the AFM interlayer interaction energy, we pick $J^{a b}=-3.5,-3.0,-2.5,-2.4,-2.3,-2.0$ to have the condition $J^{a b}<0$ with $L_{a}=L_{b}=4$, $h=1.0$. Figure $4 \mathrm{a}$ and $\mathrm{b}$ describes the two 


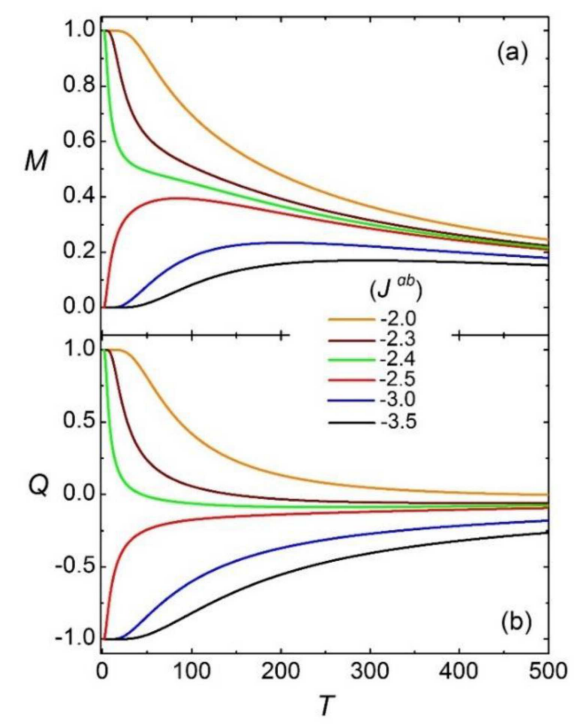

Fig. 4. (a) The same as Fig. 3e but for $h=1$, (b) the same as Fig. 3f but for $h=1$. Note that $J^{a b}<0$.

saturation values of magnetization $(M=0.0,1.0)$ and short-range order parameter $(Q=-1.0,1.0)$ to appear near the zero temperature. Meanwhile, each of $M$ and $Q$ curves has different behaviors with the increasing $T$. For example, $M$ reaches a maximum value as the temperature grows with $J^{a b}$ ranging from -3.5 to -2.5 . These maxima shift towards a lower temperature as $J^{a b}$ grows, as can be seen from the black, blue and red colored curves corresponding to $J^{a b}=-3.5,-3.0$ and -2.5 , respectively. Moreover, $M$ vs. $T$ and $Q$ vs. $T$ curves decrease with the increasing temperature in the $J^{a b}$ range from -2.4 to -2.0 . These interesting curves are attributed to the compensation between the interlayer exchange coupling $J^{a b}$ and temperature. Also, similar calculations can be carried out using different values of couplings $J_{C}^{a}\left(J_{C S}^{a}\right)$ and $J_{C}^{b}$ $\left(J_{C S}^{b}\right)$. We compare the above results (except for the short-range ordering) with the available theoretical works [42]. We find that some of the magnetization curves are very similar to those for a bilayer graphene-like structure in a longitudinal magnetic field with the compensation between the crystalfield and temperature observed by Wu et al. [42].

The $J^{a b}$ dependence of $M$ using different layer sizes $\left(L_{a}, L_{b}\right)$, temperatures $T$ and magnetic fields $h$ is shown in Fig. $5 \mathrm{a}-\mathrm{c}$, respectively. One can see in Fig. 5a that magnetization shifts to the left as the increase of a layer size. In general, $M$ curves range from 0.0 to 1.0 with increasing of $J^{a b}$. This behavior is also consistent with the general behavior in accord with the size. In Fig. 5b, we represent $M$ vs. $J^{a b}$ curves which are obtained at several different temperature values. For any temperature, the stable branches of $M$ become saturated as $M \rightarrow 1.0$ while $J^{a b}$ increases. It is determined that magnetization increases while the $J^{a b}$ increases and the

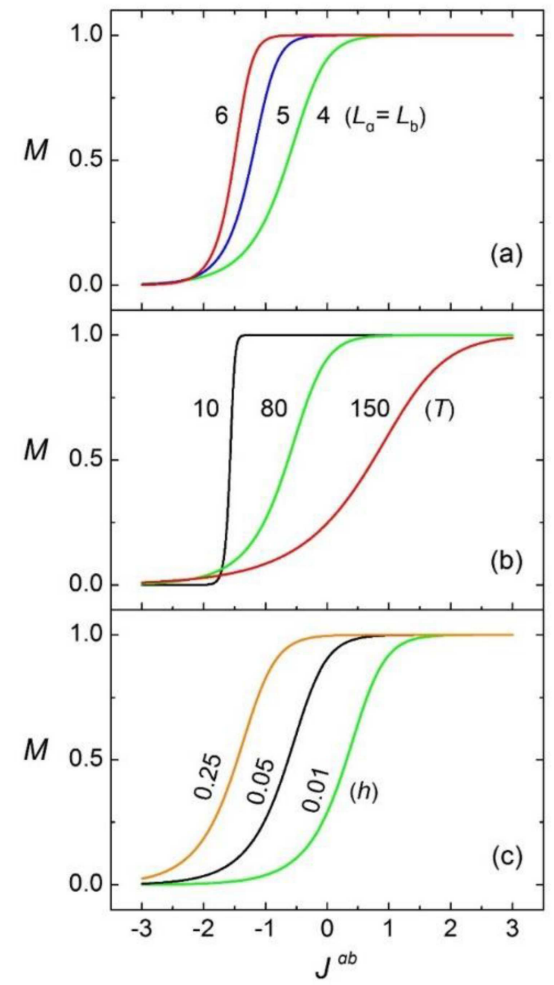

Fig. 5. (a) Interlayer coupling $J^{a b}$ dependence of $M$ at various layer sizes for $h=0.05$ and $T=80$, (b) the same as (a) but at different temperatures for $L_{a}=L_{b}=4$, (c) the same as (b) but at different field values for $T=80$.

temperature decreases at the same layer sizes. The magnetization curve of the spin- $1 / 2$ Ising bilayer still exhibits zero magnetization plateau but a continuous magnetization jump persists at low enough temperatures. It actually turns out that the size of a continuous magnetization jump is just gradually suppressed with increasing temperature. The $J^{a b}$ development of $M$ is determined to exhibit almost the same behavior in Fig. $5 \mathrm{c}$. The $J^{a b}$ evolutions of $M$ are compatible in itself according to $h=0.01,0.05$ and 0.25 . The $M$ values tend to shift to the left with the increasing magnetic field values.

In Figs. 6 and 7, magnetization $M$ as a function of the magnetic field is given. The isothermal magnetic field dependence of $M$ is a paramagnetic-type behavior for the positive and negative values of $J^{a b}$ and they are presented in Fig. 6a and b, respectively. Figure 6 a shows that the $M$ vs. $h$ curves strongly depend on the temperature. They change sharply around $h=0$ and become steeper when $T$ decreases and magnetization of the spin- $1 / 2$ Ising square lattice bilayer exhibits a zero-magnetization plateau. These results are valid for the particular case $J^{a b}=1$ which can be in the range of the localized-magnon effect $[40,54,55]$. Besides, the curves presented in Fig. 6a provide a convincing evidence that a description based on an effective Ising ferromagnet on the square lattice is faithful up to moderate temperatures with $T \leq 600$. On the other 


\section{Ö. Görgülüer et al.}

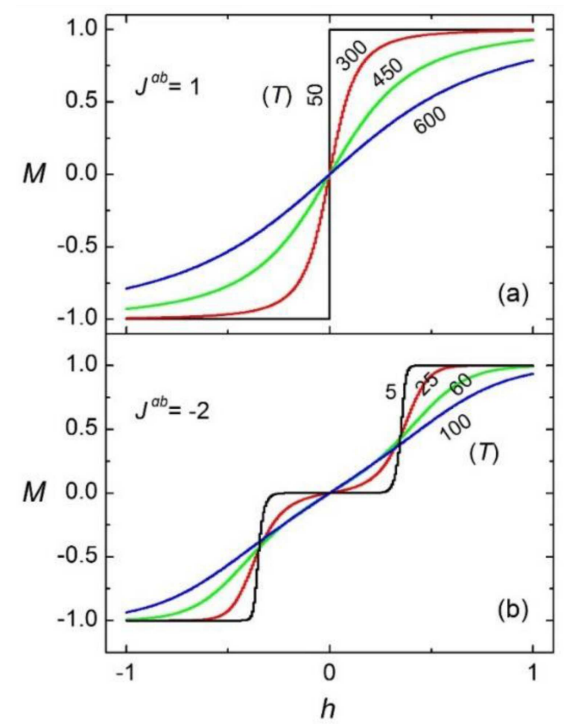

Fig. 6. (a) Magnetic field $h$ dependence of magnetization at various temperatures for the FM interlayer coupling with $J^{a b}=1.0$, (b) the same as (a) but for the AFM coupling with $J^{a b}=-2.0$. Note that $L_{a}=L_{b}=5$.

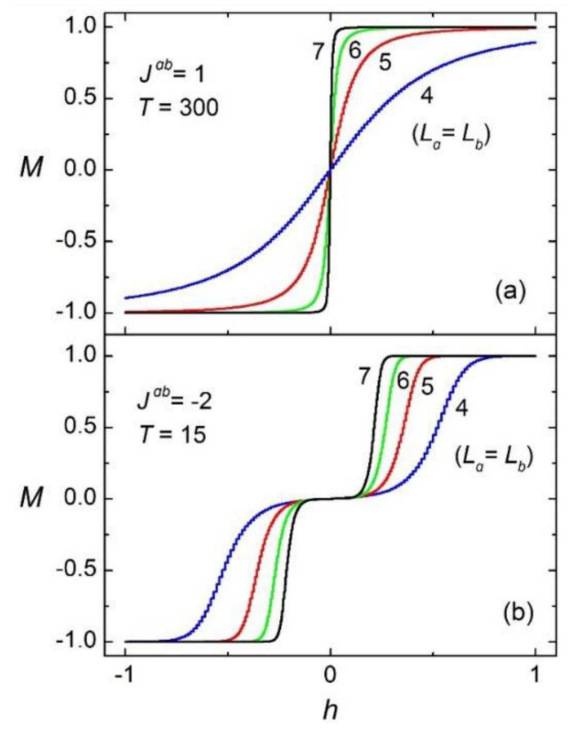

Fig. 7. (a) Magnetic field $h$ dependence of magnetization at various layer sizes for $J^{a b}=1, T=300$, (b) the same as (a) but for $J^{a b}=-2, T=15$.

hand, the $M-h$ curves using $J^{a b}=-2$ transform to the typical stepwise two isothermal magnetization curves, as seen in Fig. 6b. In other words, we observe that at low enough temperatures there exists an intermediate one-half magnetization plateau besides two full saturation plateaus. This stepwise magnetization curve with intermediate plateaus at zero of the saturation magnetization is gradually smeared out with increasing temperature. It is also noteworthy that these results are in agreement with the previous results obtained from the numerical studies of the bilayer Ising model on various

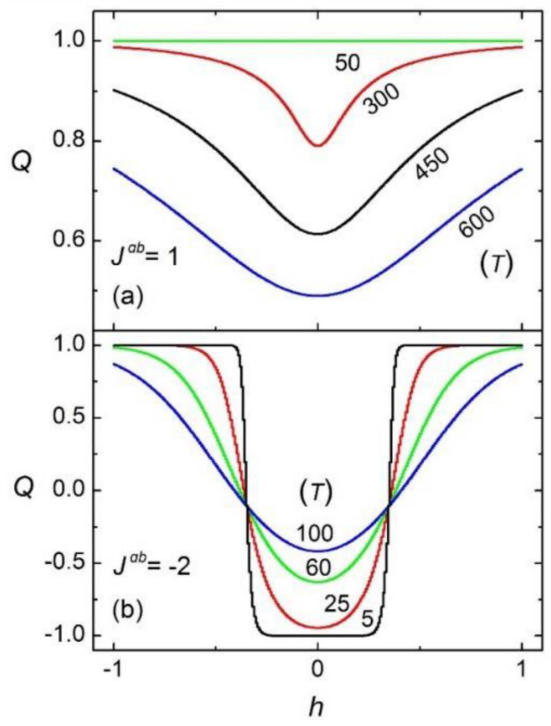

Fig. 8. (a) The same as Fig. 6a but for $Q$, (b) the same as Fig. $6 \mathrm{~b}$ but for $Q$.

lattice geometries including interlayer frustration and the case with a coupling with external magnetic field [37, 40, 41]. In addition, we have also obtained the $M-h$ curves using different layer sizes. According to Fig. 7a, we have observed almost the same behavior as in Fig. 6a. However, the $M-h$ curves in Fig. $7 \mathrm{~b}$ evolve into the smooth stepwise magnetization curve with a decreasing size of the layers. The origin of the typical stepwise/smooth stepwise transition in the bilayer system is related to the interlayer exchange coupling $J^{a b}$, the layer size and temperature.

It is also interesting to show the short-range order parameter $Q$ plotted as a function of the magnetic field for the same temperatures and the layer sizes used in the previous two figures. The calculated results corresponding to Fig. 6 are given in Fig. 8 . One can see from this figure that a short-range order parameter is independent of the orientation of the magnetic field, $Q(-h)=Q(h)$, which is in harmony with the corresponding bulk model [56]. Temperature-dependent minima (or pit) at $h=0$ are observed in the $Q>0$ region when $J^{a b}=1$ while this minima disappear and become an intermediate plateau at very low temperatures in the regime $Q<0$ for $J^{a b}=-2$. The depth of the pit for $J^{a b}=1$ is proportional to temperature while it is inversely proportional to $T$, as illustrated in Fig. $8 \mathrm{a}$ and b, respectively. It is also seen from Fig. $8 \mathrm{~b}$ that the plateau (whose width becomes larger as $T \rightarrow 0$ ) evolves into a soft pit as the temperature increases.

Another aspect of these systems is given in Fig. 9, where the corresponding $Q$ vs. $h$ curves are drawn using various layer sizes at fixed temperatures. Our results for $J^{a b}=1$ using $T=300$ and $J^{a b}=-2$ using $T=15$ are presented in Fig. 9a and b, respectively. The intermediate plateau at $Q=-1$ is also observed for all layer sizes when $J^{a b}=-2$ while 


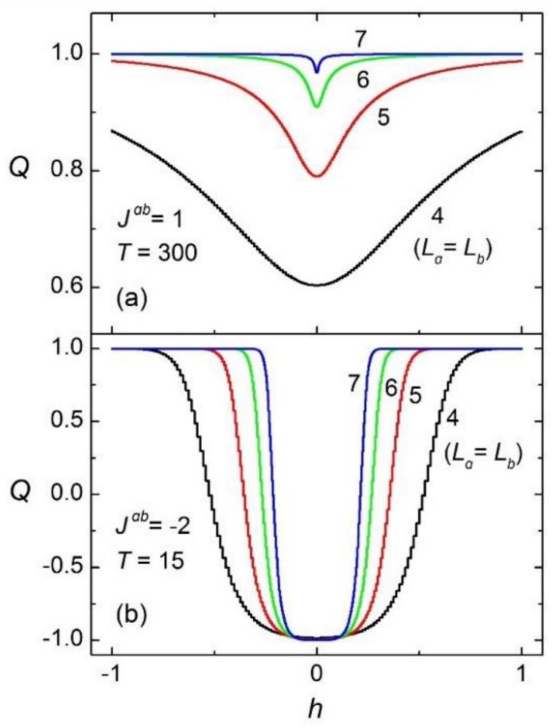

Fig. 9. (a) The same as Fig. 7a but for $Q$, (b) the same as Fig. $7 \mathrm{~b}$ but for $Q$.

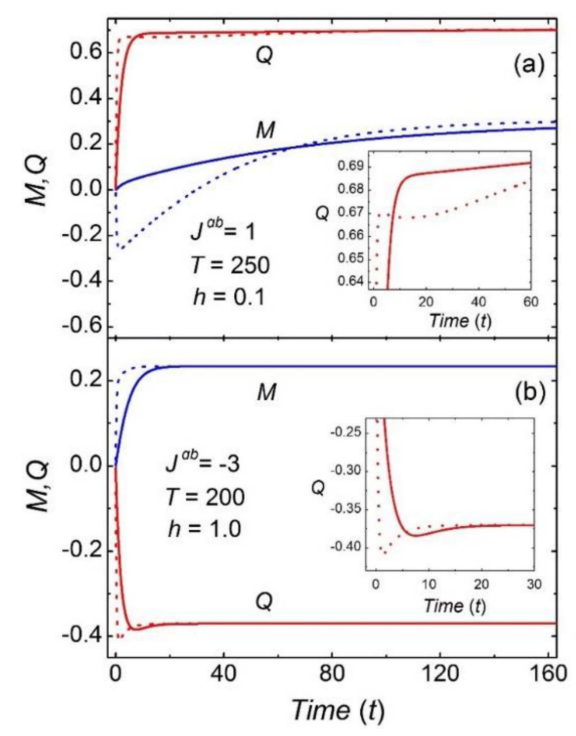

Fig. 10. (a) Relaxation curves of the order parameters $M$ and $Q$ for two different sets of values of the rate constants: $k_{1}=k_{2}=1$ (solid) and $k_{1}=1$ and $k_{2}=10$ (dotted); $J^{a b}=1.0, T=250, h=0.1$. (b) The same as (a) but for $J^{a b}=-3.0, T=200$, $h=1.0$.

this plateau disappears and becomes a minimum for $J^{a b}=1$ in the middle parts of Fig. 9a and b. In this work, the plateau and pit properties in $Q-h$ curves are attributed to the shape anisotropy in the square bilayer system.

Finally, we present the time evolution of the spin system in Figs. 10 and 11. Figure 10a and b shows the relaxation curves of $M$ and $Q$ for the selected FM and AFM interlayer interaction energies with $J^{a b}=1$ and $J^{a b}=-3$, respectively. In the figures, the solid curves are for $k_{1}=k_{2}=1$ and the dotted curves are for $k_{1}=1, k_{2}=10$. If the initial

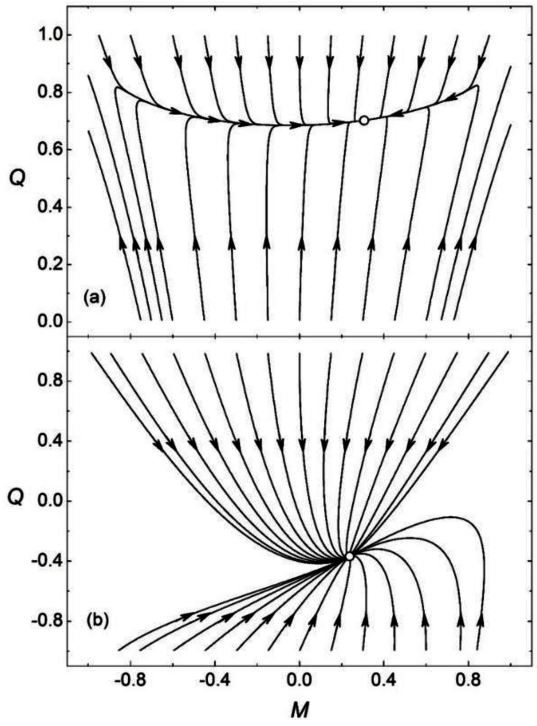

Fig. 11. Flow diagram of the bilayer system at $L_{a}=L_{b}=4$. The open circle indicates the stable state: (a) with $T=250, h=0.1, J^{a b}=1.0$ and (b) with $T=200, h=1.0, J^{a b}=-3.0$.

conditions are chosen near to the point of the total disorder $(M \approx Q \approx 0)$ when $T=200$ and 250 , the system relaxes into ordered states as the time progresses. One can see from the blue- and red-colored curves that the relaxation time for the long-range ordering is much longer than for the short-range ordering in the FM case (see Fig. 10a), whereas in the AFM case they are very close to each other (Fig. 10b). We have also found that increasing the value of $k_{2}$ leads to the speeding up of the whole relaxation process as can be seen by comparing the dotted and solid curves in Fig. 10. The reason for choosing $k_{2}>k_{1}$ is that most spin systems have a shorter relaxation time for a rotation than for a transition [57, 58]. In this case, an "overshooting" phenomenon becomes more apparent, as can be seen in the insets of Fig. 10a and b. The above relaxation results can also be observed using flow diagrams (FDs) in a two-dimensional $M-Q$ phase space describing the solutions of the dynamic equations. For the FDs, the system of equations (for the nonequilibrium case) required more than two initial values of $M$ and $Q$ in the PA method. By starting with the initial values close to the boundary, as time progresses in some given small steps, the values of $M$ and $Q$ are computed and the point referring to them also moves in the plane, as shown in Fig. 11a and b for $J^{a b}=1$ and $J^{a b}=-3$, respectively. The crucial points in the calculated FDs correspond to the stable fixed points. The coordinates of the fixed points in the diagrams are $M=0.3$, $Q=0.7$ in Fig. 11a and $M=0.23, Q=-0.37$ in Fig. 11b. The open circles correspond to the stable equilibrium solution which refers to the lowest minimum of the free energy. These are the solutions of the equilibrium case equations that can be obtained by using the iterations method. 


\section{Conclusion}

In this study, the pair approximation and the path probability method of Kikuchi have been applied to study the ferromagnetism of a square lattice bilayer system within an Ising model. The temperature, magnetic field, interlayer coupling and time evolutions of the long- and short-range order parameters have been determined. From these evolutions, the most relevant physical parameters responsible for ferromagnetism have been obtained. One of these parameters is the second-order phase transition temperatures. Also, for the first-order phase transition, both the biquadratic exchange coupling and the single-ion anisotropy constant must be at the same time in systems (see [50] for details). According to these results, it is concluded that the first and second order phase transitions are to be seen in nanodimensional structures in the presence of bilinear interactions, biquadratic interaction and single ion anisotropy. With the nanosized $C S$-type bilayers on the square lattice, we have observed that the main characteristics of magnetic behavior are related to the layer size and interlayer exchange energy coupling $J^{a b}$. First, we showed that the introduction of the interlayer exchange coupling affects the values of critical temperatures in the absence of a magnetic field. Similarly, these temperatures depend explicitly on the layer size as well as the exchange energy across a $C S$ interface in each layer. Second, we have been interested in the computations of both order parameters with a nonvanishing magnetic field and concluded that these quantities are shifted to higher temperatures, with the presence of a magnetic field $h$. There are additional remarkable properties of $M$ vs. $h$ and $Q$ vs. $h$ plots which are worth mentioning. At a lower temperature regime, the isothermal magnetization exhibits a stepwise PM type behavior while the short-range order shows a plateau at $Q=-1$ around $h=0$. These properties also depend on the layer size and $J^{a b}$. We studied the relaxations of $M$ and $Q$ towards stable equilibrium states. It becomes obvious that, for positive $J^{a b}$ but small $h$, relaxation time for $M$ is larger than for $Q$. For negative values of $J^{a b}$ and higher values of $h$, the difference between these parameters vanishes. We have shown that dynamics is governed by two statistical rate constants via the relaxation curves and FDs in the two-dimensional $M-Q$ phase space.

The investigation of magnetic properties of the $C S$-type bilayer system revealed behaviors in harmony with some of the earlier works on the same topic. For instance, magnetization results obtained from the PA technique also have the same aspect as those obtained from the Monte Carlo study of an Ising nanoisland with a bilayer graphene-like structure in a longitudinal magnetic field. We expect to extend the study to other bilayer systems on different lattice structures, like the bilayer graphene-like structure, exploiting the richness of the PA technique to obtain the complete phase diagrams. It has been found that the anisotropy causes the formation of plateaus and pits in short-range order properties. Finally, as for the short-range ordering results, we also remark that this PA static and PPM dynamic studies have not been previously considered in any investigation on the bilayer systems in the literature, to the best of our knowledge. We hope that our detailed theoretical results will stimulate further work, especially the study of the time evolutions of other layered ferromagnets.

\section{References}

[1] R.E. Camley, J. Barnaś, Phys. Rev. Lett. 63, 664 (1989).

[2] P.M. Levy, S. Zhang, A. Fert, Phys. Rev. Lett. 65, 1643 (1990).

[3] M. Akabori, S. Hidaka, H. Iwase, S. Yamada, U. Ekenberg, J. Appl. Phys. 112, 113711 (2012).

[4] J. Barnaś, Acta Phys. Pol. A 85, 165 (1994).

[5] F. Stobiecki, T. Stobiecki, Acta Phys. Pol. A 102, 95 (2002).

[6] H.W. Kunert, J. Barnaś, Acta Phys. Pol. A 132, 124 (2017).

[7] P.L. Hansen, J. Lemmich, J.H. Ipsen, O.G. Mouritsen, J. Statist. Phys. 73, 723 (1993).

[8] T. Horiguchi, N. Tsushima, Physica A 238, 295 (1997).

[9] A. Lipowski, Physica A 250, 373 (1998).

[10] T. Kaneyoshi, J. Magn. Magn. Mater. 226-230, 1746 (2001).

[11] Z.B. Li, Z. Shuai, Q. Wang, H.J. Luo, L. Schülke, J. Phys. A Math. Gen. 34, 6069 (2001).

[12] H. Kim, J. Kor. Phys. Soc. 38, 435 (2001).

[13] B. Mirza, T. Mardani, Eur. Phys. J. B 34 , 321 (2003).

[14] M. Ghaemi, B. Mirza, G. Parsafar, J. Theor. Comp. Chem. 3, 217 (2004).

[15] J.L. Monroe, Physica A 335, 563 (2004).

[16] E. Albayrak, T. Bulut, J. Magn. Magn. Mater. 316, 81 (2007).

[17] T. Balcerzak, I. Łużniak, Physica A 388, 357 (2009).

[18] O. Derzhko, T. Krokhmalskii, J. Richter, Phys. Rev. B 82, 214412 (2010).

[19] K. Szałowski, T. Balcerzak, Physica A 391 , 2197 (2012).

[20] T. Balcerzak, K. Szałowski, Physica A 395, 183 (2014).

[21] E. Kantar, M. Ertaş, Solid State Commun. 188, 71 (2014). 
[22] E. Kantar, J. Supercond. Nov. Magn. 28, 3387 (2015).

[23] E. Kantar, Phase Transit. 89, 971 (2016).

[24] E. Kantar, Mod. Phys. Lett. B 30, 1650295 (2016).

[25] M. Žukovič, A. Bobák, Phys. Lett. A 380 , 1087 (2016).

[26] R. Masrour, A. Jabar, A. Benyoussef, M. Hamedoun, J. Magn. Magn. Mater. 401, 700 (2016).

[27] E. Albayrak, A. Yigit, Acta Phys. Pol. A 116, 127 (2009).

[28] M. Žukovič, Y. Tomita, Y. Kamiya, Phys. Rev. E 96, 012145 (2017).

[29] R. Masrour, A. Jabar, J. Magn. Magn. Mater. 426, 225 (2017).

[30] A.-B. Guo, W. Jiang, N. Zhang, Physica B 513, 21 (2017).

[31] I.J.L. Diaz, N.S. Branco, Physica A 468, 158 (2017).

[32] D. Lv, W. Wang, J.-P. Liu, D.-Q. Guo, S.-X. Li, J. Magn. Magn. Mater. 465, 348 (2018).

[33] M. Keskin, M. Ertaş, Physica A 496, 79 (2018).

[34] M. Ertaş, Physica B 550, 154 (2018).

[35] M. Ertaş, M. Batı, Ü. Temizer, Chin. J. Phys. 56, 807 (2018).

[36] Y. Yüksel, Eur. Phys. J. B 91, 243 (2018).

[37] F.A.G. Albarracín, H.D. Rosales, P. Serra, Phys. Rev. E 98, 012139 (2018).

[38] T. Kaneyoshi, J. Phys. Chem. Solids 119, 202 (2018).

[39] T. Kaneyoshi, J. Supercond. Nov. Magn. 31, 3331 (2018).

[40] K. Karl'ová, J. Strečka, Solid State Commun. 281, 31 (2018).

[41] W. Wang, Q. Li, M.-Z. Wang, Y. Ma, A.-B. Guo, T. Huang, Physica E 111, 63 (2019).
[42] H.-J. Wu, W. Wang, F. Wang, B.-C. Li, Q. Li, J.-H. Xu, J. Phys. Chem. Solids 136, 109174 (2020).

[43] R. Kikuchi, J. Chem. Phys. 60, 1071 (1974).

[44] R. Kikuchi, Suppl. Prog. Theor. Phys. 35, 1 (1966).

[45] P.H.E. Meijer, M. Keskin, E. Bodegom, J. Stat. Phys. 45, 215 (1986).

[46] M. Keskin, P.H.E. Meijer, J. Chem. Phys. 85, 7324 (1986).

[47] A. Erdinç, M. Keskin, Physica A 307, 453 (2002).

[48] M. Keskin, A. Erdinç, J. Magn. Magn. Mater. 283, 392 (2004).

[49] O. Yalçın, R. Erdem, S. Övünç, Acta Phys. Pol. A 114, 835 (2008).

[50] O. Yalçın, R. Erdem, S. Özüm, J. Appl. Phys. 115, 054316 (2014).

[51] S. Özüm, O. Yalçın, R. Erdem, H. Bayrakdar, H.N. Eker, J. Magn. Magn. Mater. 373, 217 (2015).

[52] O. Yalçın, R. Erdem, S. Özüm, Z. Demir, J. Magn. Magn. Mater. 389, 120 (2015).

[53] R. Erdem, O. Yalçın, S. Özüm, N. Çiftçi, Adv. Cond. Matter. Phys. 2016, 6563274 (2016).

[54] J. Richter, Low. Temp. Phys. 31, 695 (2005).

[55] J. Strečka, K. Karl'ová, V. Baliha, O. Derzhko, Phys. Rev. B 98, 174426 (2018).

[56] R. Erdem, Physica A 526, 121173 (2019).

[57] M. Keskin, R. Erdem, J. Statist. Phys. 89, 1035 (1997).

[58] R. Erdem, S. Özüm, Mod. Phys. Lett. B 33, 1950258 (2019). 